\title{
Hypoglycemic effects of esculeoside $A$ are mediated via activation of AMPK and upregulation of IRS-1
}

\author{
Ziming Yang ${ }^{1,2}$, Li Zhang ${ }^{1,2}$, Jinglei Liu ${ }^{1,2}$, Fenglai Lu ${ }^{1,2}$, Lei Wang ${ }^{1,2}$, Yueyuan Chen ${ }^{1,2^{*}}$ and Dianpeng Li $i^{1,2^{*}}$
}

\begin{abstract}
Background: Tomato fruit (Lycopersicon esculentum Mill.) has been suggested to be useful for the prevention of diabetes. Esculeoside A is the main saponin compounds in tomatoes. This study investigated the hypoglycemic effects and the underlying mechanism of esculeoside A in C57BLKS/Leprdb ( $d b / d b)$ mice.

Methods: Wild-type C57BLKS ( $d b / d m)$ mice were used in the $d b / d m$ mouse group and $d b / d b$ mice were randomly divided into 2 groups: untreated and treated $d b / d b$ mouse groups. Esculeoside A (100 mg/kg) was administered by gavage for 56 days to the treated $d b / d b$ mouse group. Distilled water was administered to the $d b / d m$ mouse group and the untreated $d b / d b$ mouse group. The blood and liver biochemical parameters and the expression of liver insulin signaling-related proteins were examined.

Results: The results showed that esculeoside A reduced the fasting blood glucose (FBG) levels and improved the glucose tolerance. Further investigation revealed that hepatic protein expressions of total AMP-activated protein kinase (T-AMPK), phosphorylated AMP-activated protein kinase (p-AMPK), insulin receptor substrate-1 (IRS-1), and glucokinase (GCK) were significantly upregulated after esculeoside A treatment. In contrast, the hepatic protein expression of phosphoenolpyruvate carboxykinase (PEPCK) was significantly downregulated by esculeoside A treatment.

Conclusion: These findings suggested that esculeoside A has a potential of alleviating the metabolic abnormalities in $d b / d b$ mice via regulation of AMPK/IRS-1 pathway. Our findings supported a possible application of esculeoside A as a functional supplement for diabetes treatment.
\end{abstract}

Keywords: Esculeoside a, db/db mice, AMPK, IRS-1, Liver glucose metabolism

\section{Background}

Type 2 diabetes mellitus (T2DM) is a common metabolic disease worldwide. Amidst the worldwide epidemic of T2DM, 522 million people are estimated to suffer from T2DM by 2030 [1]. The increased incidence of T2DM has significantly increased the risk of associated complications, thereby reducing quality of life and increasing mortality. The patients with T2DM are prone to microvascular complications and macrovascular diseases, such as diabetic nephropathy, diabetic neuropathy, diabetic retinopathy, stroke, atherosclerosis, and hypertension $[2,3]$. The basic

\footnotetext{
* Correspondence: 18907830419@163.com; phytoldp@hotmail.com ${ }^{1}$ Guangxi Key Laboratory of Functional Phytochemicals Research and Utilization, Institute of Botany, Guangxi Zhuang Autonomous Region and Chinese Academy of Sciences, Guilin 541006, China

Full list of author information is available at the end of the article
}

pathogenesis of T2DM is characterized by hyperglycemia, relative impairment in insulin secretion, and insulin resistance [4]. In particular, insulin resistance is regarded as a major contributor in the occurrence and development of T2DM [5]. The liver is very important for metabolic homeostasis, and controls glucose utilization and production. It is a key organ for insulin activity. Insulin regulates lipogenesis and restrains gluconeogenesis in the liver. Insulin resistance leads to abnormalities in hepatic glucose output, and leads to hyperglycemia, which results in further worsening of the hepatic insulin insensitivity [6]. Insulin triggers series of signaling cascades at the cellular level, and insulin receptor substrate-1 (IRS-1) is crucial in this process. IRS-1 has also been linked to the treatment of hepatic insulin resistance [7]. Energy metabolism

(c) The Author(s). 2019 Open Access This article is distributed under the terms of the Creative Commons Attribution 4.0 International License (http://creativecommons.org/licenses/by/4.0/), which permits unrestricted use, distribution, and reproduction in any medium, provided you give appropriate credit to the original author(s) and the source, provide a link to the Creative Commons license, and indicate if changes were made. The Creative Commons Public Domain Dedication waiver (http://creativecommons.org/publicdomain/zero/1.0/) applies to the data made available in this article, unless otherwise stated. 
imbalance is a vital problem during T2DM. AMPactivated protein kinase (AMPK) is critical in regulating energy storage and utilization [8].

T2DM is often closely associated with dietary habits and lifestyle. With the socioeconomic development and changes in people's diets, it is estimated that the prevalence of T2DM will increase tremendously over the next few decades. As a result, its high prevalence will cause great pressure on families and society, and it is important to find effective means to prevent the occurrence of T2DM.

Tomato is one of the most frequently consumed vegetables, and it has been suggested to be useful in preventing diabetes, obesity, coronary heart disease, hypertension, and other chronic diseases [9]. Studies have shown that lycopene, a component of tomato extract, can reduce blood sugar, improve lipid metabolism, and ameliorate diabetic nephropathy [10]. Esculeoside A was the first compound isolated from the cherry tomatoes (Lycopersicon esculentum Mill.); the quantity of esculeoside A was four times higher than that of lycopene in tomatoes [11]. Further investigation indicated that esculeoside A and its aglycone esculeogenin A could inhibit foam cell formation in vitro, reduce blood lipid levels, and inhibit the formation of atherosclerotic plaques in vivo [11].

Our previous studies have shown that the tomato saponin crude extract (TSCE) exhibited hypoglycemic effects in $d b / d b$ mice (unpublished). To identify the bioactive components of TSCE, we previously analyzed the content of esculeoside A in cherry tomatoes and TSCE $[12,13]$. Esculeoside A is a major constituent of TSCE (approximately $130 \mathrm{mg} / \mathrm{g}$ of TSCE), and accounts for $0.021 \%$ of dry weight of cherry tomatoes (Lycopersicon esculentum). Although previous studies have already shown that esculeoside A may alleviate lipid metabolic disorders [11], whether esculeoside A exhibits hypoglycemic effect during the treatment of diabetes is still unclear. The $d b / d b$ mice represent a type of spontaneous obese diabetic mouse model [14], while the glucose and lipid metabolism disorders in these mice are consistent with human T2DM [15, 16]. In the present study, we analyzed the hypoglycemic effects of esculeoside A isolated from Lycopersicon esculentum in $d b / d b$ mice, and investigated the possible mechanism of its action.

\section{Methods}

\section{Chemicals and reagents}

The serum total cholesterol (TC) and triglyceride (TG) kit were purchased from Changchun Huili Co., Ltd. (Changchun, China). ELISA kits for serum insulin (INS), tumor necrosis factor (TNF- $\alpha$ ), interleukin-6 (IL-6), and interleukin-1 $\beta$; ELISA kits for tissue TNF- $\alpha$, IL-6, IL-1 $\beta$; and kits for superoxide dismutase (SOD), malondialdehyde (MDA), serum alanine aminotransferases (ALT), and aspartate aminotransferases (AST) were purchased from Nanjing Jiancheng Bioengineering Institute (Nanjing, China). The tissue TC and TG assay kits were purchased from Beijing ApplyGen Technologies Inc. (Beijing, China). The total protein extraction kit was purchased from Nanjing KeyGen Biological Technology Co., Ltd. (Nanjing, China). The BCA protein quantitation kit was purchased from Biyuntian Co., Ltd. (Wuhan, China). The primary anti-p-AMPK (sc-98,485), anti-T-AMPK (sc-74,461), antiIRS-1 (sc-559), anti-phosphoenolpyruvate carboxykinase (PEPCK) (sc-32,879), anti-glucokinase (GCK) (sc-7908), and anti- $\beta$-actin $(\mathrm{sc}-19,879)$ antibodies were purchased from Santa Cruz Biotechnology, Inc. (Dallas, TX, USA). The ECL chemiluminescence kit was purchased from Pierce Manufacturing, Inc. (Bradenton, FL, USA). The NC membranes were purchased from Nanjing Madite Co. (Nanjing, China). The 30\% acrylamide, TEMED, XT sample buffer (\#161-0791) and XT reducing agent (\#1610792) were purchased from Bio-Rad (Hercules, CA, USA).

\section{Extraction of the TSCE}

Fresh cherry tomatoes (Lycopersicon esculentum Mill.) were acquired from the Guangxi Zhuang Valley Agricultural Science and Technology Co., Ltd. (Baise, China) in 2016, and identified by Prof. Yan Liu (Guangxi Institute of Botany). The voucher specimen (LE20160306) was deposited in the Herbarium of Guangxi Institute of Botany, China. The extraction of the TSCE from tomatoes was performed as described previously [12]. In brief, cherry tomatoes $(10 \mathrm{~kg})$ were washed and smashed into pulp. The obtained tomato juice was incubated at $50{ }^{\circ} \mathrm{C}$ for $2 \mathrm{~h}$ with a $0.5 \%$ commercial pectinase for enzymatic hydrolysis (Pectinex Ultra SP-L). The mixture was filtered through a 80-100 mesh filter cloth, and then, centrifuged at $3000 \mathrm{rpm} / \mathrm{min}$ for $10 \mathrm{~min}$. The supernatant was loaded onto a D-101 macroporous resin column. The column was first washed with water, and, then eluted with $80 \%$ ethanol. The $80 \%$ ethanol effluent was collected, and TSCE (65 g) was obtained after drying under reduced pressure.

\section{Isolation and structural characterization of esculeoside $\mathrm{A}$}

The TSCE $(10 \mathrm{~g})$ was dissolved in 30\% methanol loaded onto a HP-20ss column, and eluted with a gradient starting from $40 \%$ aq. $\mathrm{MeOH}$ to $100 \% \mathrm{MeOH}$. The $60 \%$ eluate was collected, loaded on a Sephadex LH-20 column and eluded with $30 \%$ methanol. The procedure yielded $1332 \mathrm{mg}$ of esculeoside A. The structure of esculeoside A was determined by high resolution mass spectrometry and nuclear magnetic resonance spectroscopy. Comparing the data of ${ }^{1} \mathrm{H}$ NMR, ${ }^{13} \mathrm{C}$ NMR, and high resolution mass spectrometry with reference previous study [17], the structure of esculeoside A was characterized as $(23 S, 25 S)$ - 23 - acetoxy - $5 \alpha, 22 \alpha N-3 \beta, 27$ - dihydroxyspirosolan 3 - $O$ - $\beta$ - 
lycotetraosyl 27 - $O-\beta$ - $D$ - glucopyranoside. The chemical structure of esculeoside A is shown in Fig. 1.

\section{Animals}

All animal procedures were previously approved by the Ethical Committee for Animal Research of the Guangxi Zhuang Autonomous Region and Chinese Academy of Science, Guangxi Institute of Botany. Male 4-week-old C57BLKS/Leprdb $(d b / d b)$ mice and wild-type C57BLKS $(\mathrm{db} / \mathrm{dm})$ mice were purchased from the Model Animal Research Center of Nanjing University.

\section{Experimental design}

The mice were kept at $24 \pm 2{ }^{\circ} \mathrm{C}$ and $45-50 \%$ relative humidity with a 12 -h light/12-h dark cycle. The mice were acclimated for 7 days before beginning the experiment. They were provided access to feed and water freely. Eight $d b / d m$ mice were used in the $d b / d m$ mouse group and $16 \mathrm{db} / \mathrm{db}$ mice were randomly divided into 2 groups (8 mice per group): untreated and treated $d b / d b$ mouse groups. Esculeoside A $(100 \mathrm{mg} / \mathrm{kg})$ in $0.2 \mathrm{ml} / 10 \mathrm{~g}$ was administered by gavage to the treated $d b / d b$ mouse group. As the vehicle control, distilled water was given to the $d b / d m$ mouse group and the untreated $d b / d b$ mouse group. All animals were treated once a day for a consecutive 56 days. The mice were weighted every 7 days and the treatment dosage was adjusted according to the body weight of the animals.

At the end of the 56 days, the mice were anesthetized with intraperitoneal injection of $120 \mathrm{mg} / \mathrm{kg}$ pentobarbital (manufacturer: HeFei BoMei Biotechnology Co.Ltd., lot number: 110919). After loss of consciousness, blood samples were collected from the abdominal aorta and the mice were sacrificed by cervical dislocation. Blood was placed into a sterile EP tube, centrifuged at 3500 rpm for $10 \mathrm{~min}$ at $4{ }^{\circ} \mathrm{C}$, and the serum obtained was stored at $-20^{\circ} \mathrm{C}$. Additionally, the liver were removed, and the liver index was calculated using the following formula: liver index = liver mass $(\mathrm{mg}) /$ mice body weight (g). Part of the liver was homogenized using a glass homogenizer, centrifuged at $3500 \mathrm{rpm}$ for $10 \mathrm{~min}$ at $4{ }^{\circ} \mathrm{C}$, and the supernatant was stored at $-80^{\circ} \mathrm{C}$. A defined amount of liver tissue was placed in a sterilized frozen tube and stored in liquid nitrogen for western blot analysis. The other part of the liver was stored in a sterilized frozen tube at $-80^{\circ} \mathrm{C}$ until hepatic lipid measurement.

\section{Determination of fasting blood glucose and glucose tolerance}

Fasting blood glucose (FBG) was evaluated every 7 days. After $12 \mathrm{~h}$ of fasting, $100 \mathrm{mg} / \mathrm{kg}$ esculeoside A was administered by gavage to the treated $d b / d b$ mouse group. Distilled water was given by gavage to both the $d b / d m$ mouse group and the untreated $d b / d b$ mouse group. Two hours later, a blood glucose meter and test strips were used for blood glucose (BG) measurement.

At the end of the 55th day, a glucose tolerance test was conducted. The mice were fasted and treated with esculeoside A (or water as control) as described above, and $2 \mathrm{~h}$ later, were intraperitoneally injected with $2.5 \mathrm{~g} /$ $\mathrm{kg}$ glucose. The blood glucose levels were determined at $0,0.5,1$, and $2 \mathrm{~h}$ later using a blood glucose meter.

Determination of blood and liver biochemical parameters Serum levels of TC, TG, ALT, and AST were determined using a semi-automatic biochemical analyzer according

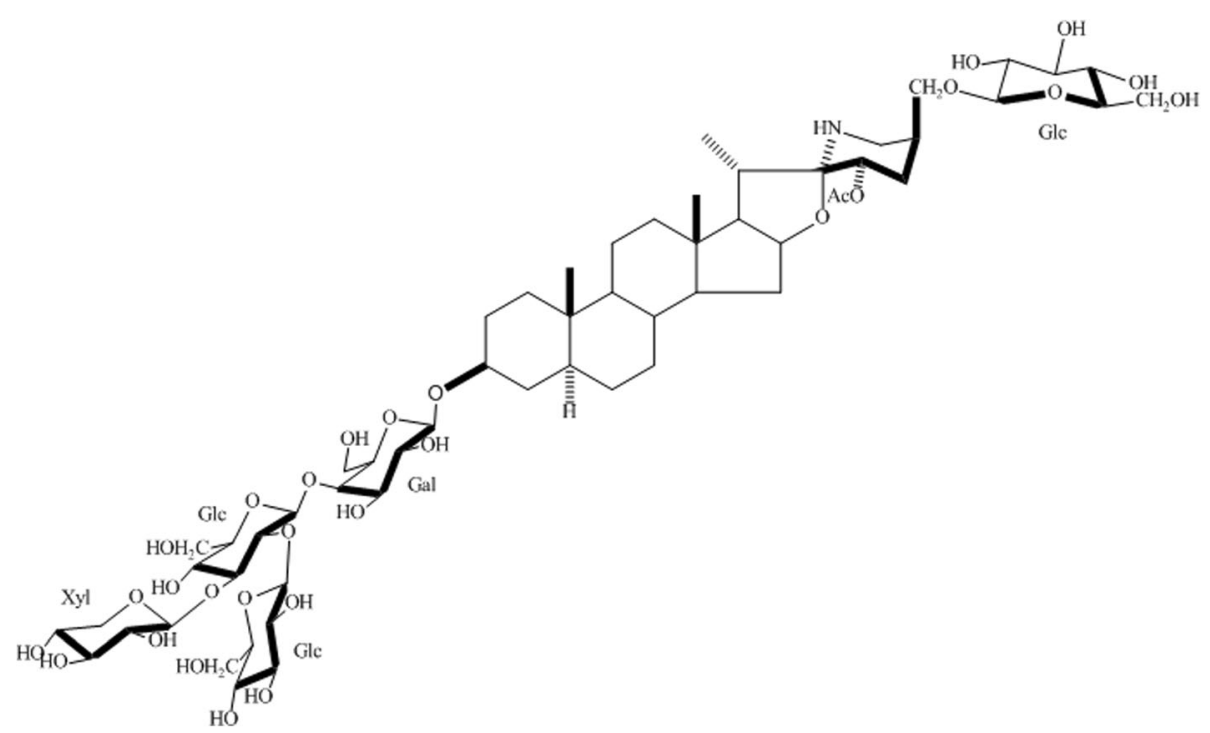

Fig. 1 Chemical structure of Esculeoside A 
to the method described by the manufacturer. Serum levels of INS were determined using an automatic microplate reader according to the manufacturer's instructions. Hepatic levels of SOD and MDA were determined using a semi-automatic biochemical analyzer according to the method described by the manufacturer. Inflammatory cytokines, including TNF- $\alpha$, IL- 6 , and IL- $1 \beta$ were determined using commercial ELISA kits. Liver TC and TG levels were determined using a semi-automatic biochemical analyzer using the tissue TC and TG commercial kit obtained from Beijing ApplyGen Technologies Inc. An accurate amount of liver tissue was weighed and $10 \mu \mathrm{L} / \mathrm{mg}$ of lysis buffer was added. The liver tissue was homogenized using a glass homogenizer, placed in a sterilized EP tube, and allowed to sit for $10 \mathrm{~min}$. The EP tube was then placed in a $70^{\circ} \mathrm{C}$ water bath for $10 \mathrm{~min}$. After cooling, the mixture was centrifuged at 2000 rpm for $5 \mathrm{~min}$, the supernatant was collected, and used for tissue TC and TG measurement $[18,19]$.

\section{Determination of hepatic insulin signaling-associated protein expression}

Liver tissue lysates were prepared using RIPA extraction buffer according to the manufacturer's instructions. A portion of the supernatant was used for protein concentration determination using the $\mathrm{BCA}$ method, and the remaining supernatant was diluted with 4-fold sample buffer, sealed, and heated at $95^{\circ} \mathrm{C}$ to denature the protein. The obtained material was then stored at $-80^{\circ} \mathrm{C}$. The protein separation gels with different concentrations were prepared according to the molecular weight of the proteins. Samples with $50 \mu \mathrm{g}$ of total protein in each sample were loaded and 6\% SDS-PAGE gel electrophoresis was carried out using a Cell electrophoresis tank. The proteins on the gel were transferred onto a membrane under a constant current of $0.32 \mathrm{~A}$ in ice water bath. After protein transfer was completed, the membrane was blocked by skim milk. Primary antibody was added and incubated overnight at $4{ }^{\circ} \mathrm{C}$. Then, secondary antibody was incubated at $37^{\circ} \mathrm{C}$ for $1 \mathrm{~h}$ and chemiluminescence determination was carried out after washing. Images were taken using a gel-imager and an enhanced chemiluminescence assay was used for the detection of protein expression. An image processing system was used for semiquantitative analysis of the target bands and a gel analysis software was used for the analysis of the average optical density of each band. The optical density value was used to represent the corresponding protein expression. The expression level of the protein of interest was expressed as a relative value by comparison with the expression level of the internal reference, $\beta$-actin [20].

\section{Statistical analysis}

Experimental data were expressed as mean values with corresponding standard errors. One-way analysis of variance (ANOVA) was used for the comparison among multiple samples. Statistical analysis was conducted using SPSS15.0 and $P<0.05$ was considered as statistically significant.

\section{Results}

Effects of Esculeoside A on body weight, FBG, and glucose tolerance in $d b / d b$ mice

The changes in the body weight can reflect the growth of the mice and may reflect adverse effects on the body. The body weights of all the mice were stable throughout the experiment (Fig. 2A). Body weights were remarkably higher in the $d b / d b$ mice than the $d b / d m$ mice. However, there was no statistically significant difference between the esculeoside A-treated and esculeoside Auntreated $d b / d b$ mice.

Although there were no significant changes in body weight in the treated $d b / d b$ mice, the FBG levels was notably decreased after treatment with esculeoside A (Fig. 2B). Reduction in FBG levels occurred after 2 weeks of the treatment, and continued to decline, while that of the control group remained stable during the course of the experiment.

The results of the intraperitoneal glucose tolerance test are shown in Fig. 2C. After intraperitoneal injection of $2.5 \mathrm{~g} / \mathrm{kg}$ glucose, the BG levels in $d b / d m$ mice started increasing and reached a peak value after $0.5 \mathrm{~h}$. Thereafter, it gradually decreased and returned to normal levels after $2 \mathrm{~h}$. After intraperitoneal injection of glucose, the BG levels in untreated $d b / d b$ mice exhibited a rapid increase. After $2 \mathrm{~h}$, the BG levels were still increasing. In contrast, the BG levels in treated $d b / d b$ mice rapidly decreased after reaching peak value at $0.5 \mathrm{~h}$. Compared with the untreated $d b / d b$ mice, the BG levels in treated $d b / d b$ mice $20.7 \%$ lower $1 \mathrm{~h}$ after glucose injection. Two hours after glucose injection, the BG levels were found to be $20.9 \%$ lower in treated $d b / d b$ mice.

\section{Serum and liver analyses 8 weeks after treatment with esculeoside $A$}

As shown in Table 1, the untreated $d b / d b$ mice showed typical type 2 diabetes characteristics, such as elevated levels of insulin and blood lipid, indicating the abnormal metabolisms of lipid as well as insulin resistance. However, esculeoside A administration in $d b / d b$ mice did not significantly affect these parameters.

Examination of the mouse liver found that the liver indices, fat content, and liver damage sensitivity indices, ALT and AST levels, were significantly elevated in the untreated $d b / d b$ mice. After esculeoside A treatment, the liver indexes were significantly decreased. Many 

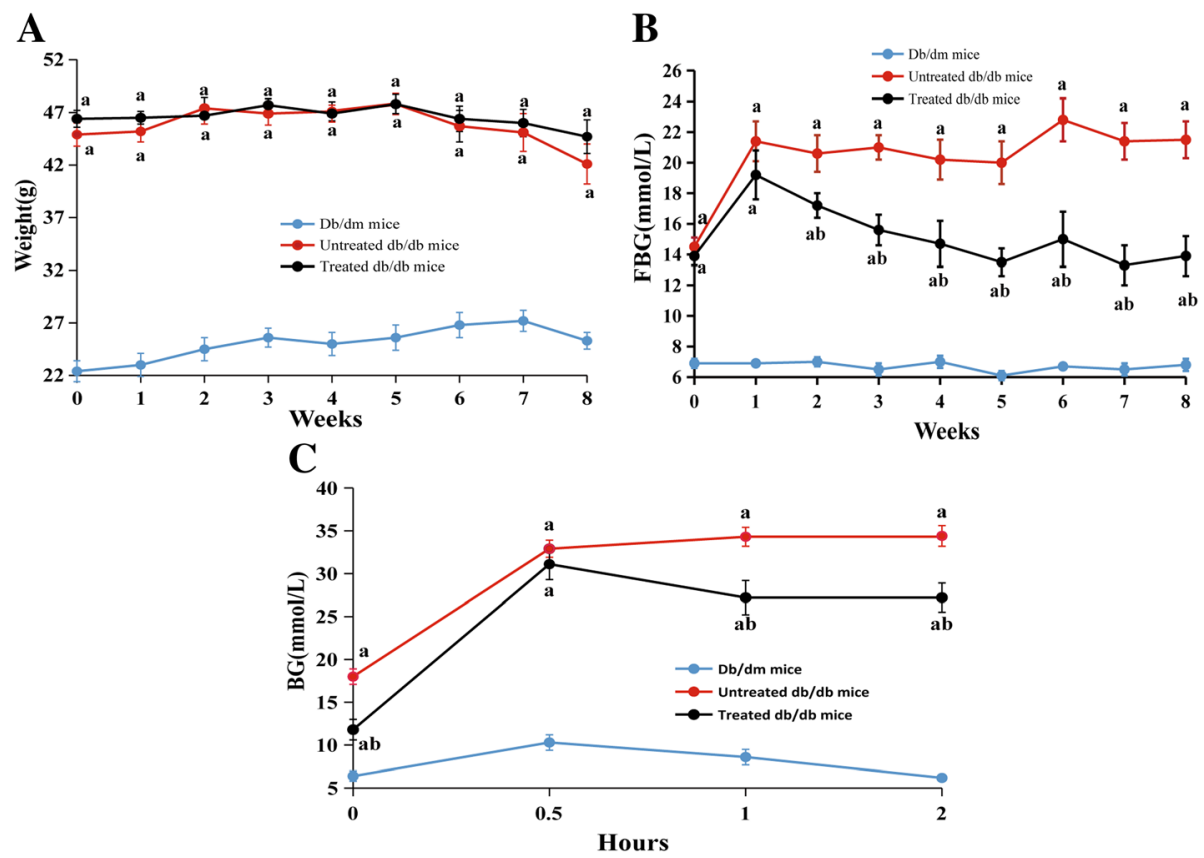

Fig. 2 Effects of Esculeoside A on body weight, FBG, and glucose tolerance in db/db mice. A Body weights, B FBG, and $\mathbf{C}$ glucose tolerance. The results are expressed as mean \pm SEM ( $n=8$ per group). Values having different superscripts are significantly different, $P<0.05$, one-way ANOVA test. a. Statistical difference compared to the $d b / d m$ mice, b. Statistical difference compared to the untreated $d b / d b$ mice

Table 1 Effects of esculeoside A on the biochemical parameters and liver index of $d b / d b$ mice

\begin{tabular}{llll}
\hline Item & Db/dm mice & Untreated $d b / d b$ mice & Treated $d b / d b$ mice \\
\hline Serum & & & $6.86 \pm 0.87^{\mathrm{a}}$ \\
TC (mmol/L) & $3.32 \pm 0.22$ & $7.05 \pm 0.38^{\mathrm{a}}$ & $1.20 \pm 0.25^{\mathrm{a}}$ \\
TG (mmol/L) & $0.96 \pm 0.08$ & $1.26 \pm 0.19^{\mathrm{a}}$ & $64.2 \pm 12.1^{\mathrm{a}}$ \\
INS (m/U/L) & $9.0 \pm 0.9$ & $70.1 \pm 9.5^{\mathrm{a}}$ & $61.3 \pm 7.2^{\mathrm{a}}$ \\
ALT (U/L) & $24.3 \pm 1.9$ & $59.2 \pm 4.8^{\mathrm{a}}$ & $81.5 \pm 9.8^{\mathrm{a}}$ \\
AST (U/L) & $49.5 \pm 3.1$ & $88.8 \pm 7.2^{\mathrm{a}}$ & $355.1 \pm 19.5^{\mathrm{b}}$ \\
TNF-a (ng/L) & $95.3 \pm 9.8$ & $390.6 \pm 23.8^{\mathrm{a}}$ & $123.8 \pm 19.2^{\mathrm{a}}$ \\
IL-6 (ng/L) & $33.5 \pm 3.6$ & $150.5 \pm 14.8^{\mathrm{a}}$ & $8.5 \pm 1.9^{\mathrm{a}}$ \\
IL-1 $\beta$ (ng/L) & $6.5 \pm 0.7$ & $11.8 \pm 1.5^{\mathrm{a}}$ & \\
Liver & & & $50.98 \pm 1.98^{\mathrm{b}}$ \\
Liver index (mg/g) & $49.58 \pm 1.14$ & $57.25 \pm 1.57^{\mathrm{a}}$ & $0.33 \pm 0.05^{\mathrm{a}}$ \\
TC (mg/mg prot) & $0.16 \pm 0.02$ & $0.31 \pm 0.04^{\mathrm{a}}$ & $5.46 \pm 0.92^{\mathrm{a}}$ \\
TG (mg//mg prot) & $1.67 \pm 0.25$ & $5.68 \pm 0.61^{\mathrm{a}}$ & $2.96 \pm 0.28^{\mathrm{b}}$ \\
TNF-a (pg/mg prot) & $2.38 \pm 0.35$ & $4.71 \pm 0.49^{\mathrm{a}}$ & $10.96 \pm 1.02^{\mathrm{a}}$ \\
IL-6 (pg/mg prot) & $3.12 \pm 0.35$ & $12.15 \pm 0.78^{\mathrm{a}}$ & $1.68 \pm 0.21^{\mathrm{a}}$ \\
IL-1 $\beta$ (pg/mg prot) & $0.77 \pm 0.09$ & $2.07 \pm 0.22^{\mathrm{a}}$ & $4.26 \pm 0.48$ \\
SOD (U/mg prot) & $4.61 \pm 0.39$ & $4.35 \pm 0.42$ & $3.11 \pm 0.31^{\mathrm{b}}$ \\
MDA(nmol/mg prot) & $2.67 \pm 0.31$ & $4.32 \pm 0.35^{\mathrm{a}}$ & \\
\hline
\end{tabular}

The results are expressed as mean \pm SEM ( $n=8$ per group). Values having different superscripts are significantly different, $P<0.05$, one-way ANOVA test. ${ }^{\mathrm{a}}$ Statistical difference compared to the $d b / d m$ mice, ${ }^{\mathrm{b}}$ Compared to the untreated $d b / d b$ mice 
inflammatory factors, such as TNF- $\alpha$, IL-6, and IL-1 $\beta$ are closely associated with liver injury. We used ELISA to detect the levels of TNF- $\alpha$, IL- 6 , and IL- $1 \beta$ in serum and liver. The results showed that they were significantly increased in untreated $d b / d b$ mice; however, after esculeoside A administration, only the levels of TNF- $\alpha$ in serum were significantly decreased. We also found the levels of hepatic MDA in untreated $d b / d b$ mice were significantly higher compared to the $d b / d m$ mice, and esculeoside A treatment significantly prevented the formation of this product of lipid peroxidation.

\section{Effects of esculeoside $A$ on the expression of proteins involved in glucose uptake}

The $d b / d b$ mice were treated with esculeoside A for 56 days, and its effects on the expressions of the proteins associated with glucose uptake were investigated (Fig. 3). After esculeoside A treatment, the hepatic proteins expressions of AMPK, p-AMPK, GCK, and IRS-1 were significantly upregulated. In contrast, the hepatic proteins expression of PEPCK was significantly downregulated after esculeoside A treatment. The hepatic AMPK, pAMPK, and IRS-1 proteins levels remained low and the hepatic PEPCK protein levels remained high in untreated $d b / d b$ mice. These data suggested that AMPK and IRS-1 pathways contribute to the esculeoside A- mediated downregulation of hepatic glucose production and increase in glucose utilization.

\section{Discussion}

In recent years, the clinical focus has switched to natural products to treat T2DM. Tomatoes have been suggested to be useful in the prevention of diabetes. We extracted the water-soluble saponin compound esculeoside A from Lycopersicon esculentum and studied its hypoglycemic effects in experimental type 2 diabetes mice model. The results demonstrated that esculeoside A possessed antihyperglycemic properties and the mechanism is promoting AMPK and IRS-1 pathways.

AMPK is considered to be an intracellular "fuel gauge" that plays a vital role in controlling the energy homoeostasis, including the regulation of lipid metabolism, glycogen metabolism, fatty acid oxidation, and BG levels $[8,21]$. AMPK can also be used as a therapeutic target for the treatment of metabolism-related diseases [22]. Several drugs have been widely reported to activate AMPK. For instance, metformin significantly enhances AMPK phosphorylation and regulates glycometabolism, and rosiglitazone reduces blood sugar levels and increases AMPK expression [23]. Previous research has shown that the expression of AMPK gene is downregulated in the $d b / d b$ mice [24], and our results were
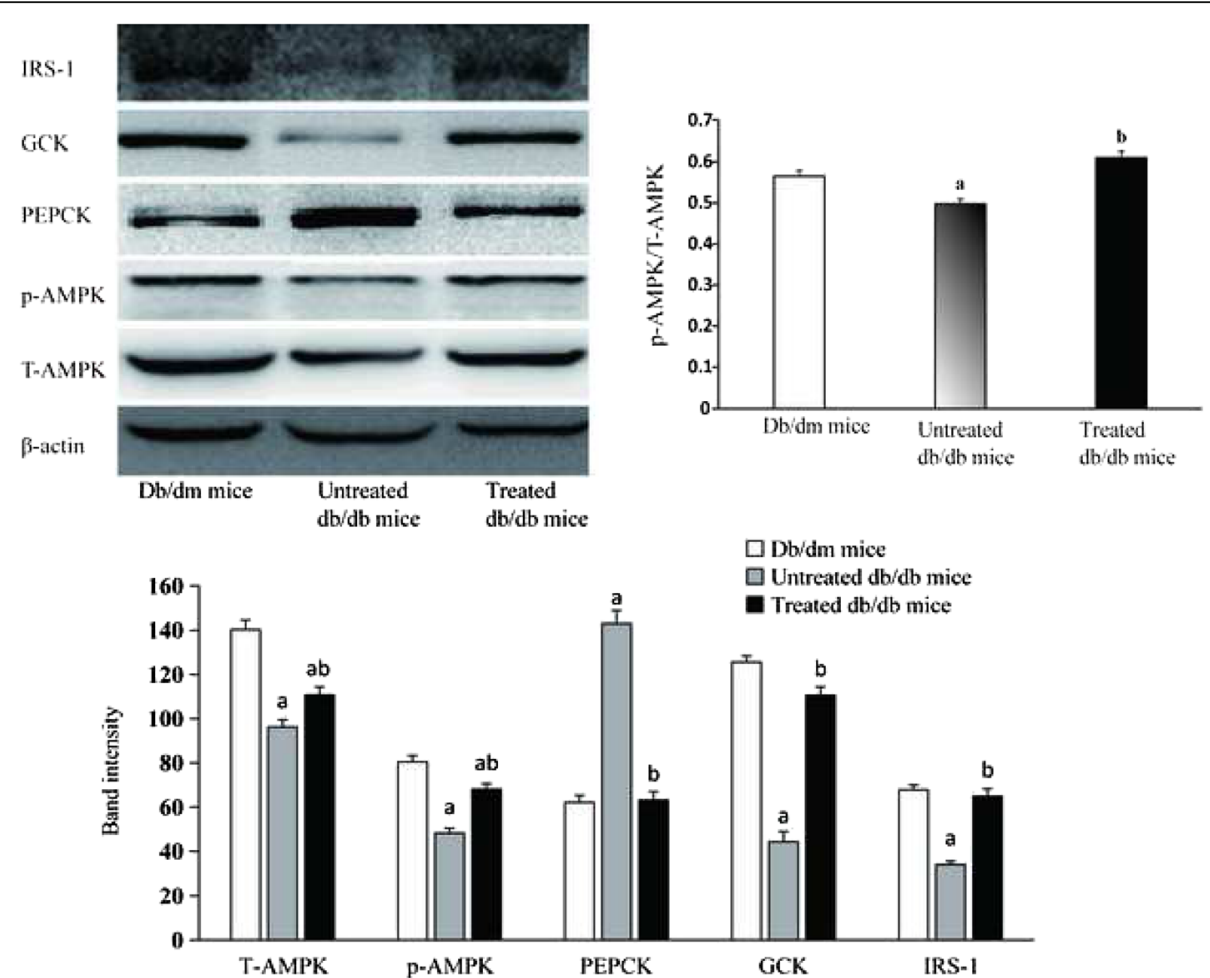

Fig. 3 Effects of esculeoside A on the proteins involved in glucose uptake pathway. Values having different superscripts are significantly different, $P<0.05$, one-way ANOVA test. a. Statistical difference compared to the $d b / d m$ mice, b. Statistical difference compared to the untreated $d b / d b$ mice 
consistent with this finding. The energy metabolism of untreated $d b / d b$ mice was impaired. Maintaining such a state for a long period of time leads to hyperglycemia, hyperlipidemia, and weight gain in untreated experimental animals. After esculeoside A treatment, we observed an upregulation in AMPK expression in the $d b / d b$ mice. The activation of AMPK inhibited two key gluconeogenic enzymes, glucose 6-phosphatase and PEPCK [25]. The major organ for gluconeogenesis is the liver, while PEPCK in liver represents a crucial rate limiting enzyme in the gluconeogenesis pathway, since its transcription level determines the rate of gluconeogenesis [26]. Previous research has indicated that downregulation of PEPCK expression causes reduction in glucose synthesis [27, 28]. In this study, we observed a downregulation of the hepatic PEPCK expression after esculeoside A treatment. This result indicated that the hepatic gluconeogenesis is significantly decreased, eventually leading to decrease BG levels in treated $d b / d b$ mice. These findings suggested that esculeoside A possessed anti-hyperglycemic properties and promoted glucose uptake by activated AMPK pathway in liver. Notably, esculeoside A treatment did not significantly alter TC and TG levels in serum or liver, and did not cause any weight loss in treated $d b / d b$ mice. However, AMPK plays important role in regulating metabolic diseases, such as obesity, diabetes mellitus, etc. It has previously been reported that AMPK also decreased the levels of glucose, cholesterol, and triglycerides, and enhanced fatty acid oxidation $[8,29]$. A possible explanation is that esculeoside A activated AMPK pathway was only partially accountable for lowering the blood glucose levels. Furthermore, after esculeoside A treatment, it only increased pAMPK levels by $33 \%$, but the hepatic PEPCK expression was decreased by $63 \%$. This result suggested that the expression of PEPCK gene might be regulated by other factors.

The $d b / d b$ mice are typical hepatic insulin-resistant mice [15]. Decreased insulin sensitivity in the liver can result in increase in glucose production leading to hyperglycemia. Insulin activates metabolism signaling pathways in cells. The signaling pathways regulated by phosphatidylinositol 3-kinase and insulin receptor substrate (IRS) play a vital role in the metabolism of insulin [7]. The two major IRS subtypes known as IRS-1 and IRS-2. They are highly expressed in mice liver. It has previously been reported that both the IRS isoforms have complementary functions in the regulation of hepatic metabolism. IRS-1 gene is more closely associated with hepatic glucose homeostasis, whereas IRS-2 gene is more closely associated with hepatic lipid homeostasis [7]. Studies have shown that Knockdown of IRS-1 gene leads to the upregulation of the expression levels of gluconeogenic enzyme and PEPCK, and the decreased IRS1 expression is also associated with decreased GCK expression and lead to increase blood glucose [30]. We showed that the hepatic expression of IRS-1 was significantly decreased in untreated $d b / d b$ mice. Abnormity in the insulin signaling pathway is thought to result in increased PEPCK expression and decreased GCK expression. After esculeoside A treatment, the expression level of IRS-1 in liver was restored. As mentioned earlier, the hepatic PEPCK expression in the treated $d b / d b$ mice was decreased. GCK is mainly expressed in the liver and catalyzes cell glucose phosphorylation, and is responsible for glucose homeostasis [31]. Loss of GCK activity leads to diabetes in humans and animals [32]. Our study showed that the expression of GCK in the treated $d b / d b$ mice was $150 \%$ higher than in the untreated $d b / d b$ mice. The hepatic PEPCK expression was decreased and GCK expression was increased, both contributing to reduction of BG levels in treated $d b / d b$ mice. Therefore, we speculated that esculeoside A could regulate the function of insulin and promote insulin signal transduction.

In the present study, we showed that glucose tolerance in untreated $d b / d b$ mice was negatively affected. Impaired glucose tolerance was mainly due to insulin resistance in muscles and fat tissues [33, 34], as characterized by reduced insulin-induced muscle and fat absorption of glucose, resulting in reduced glucose utilization and increased postprandial blood sugar. Due to the ability of esculeoside A to improve the impaired glucose tolerance in treated $d b / d b$ mice, we speculated that it is partly accountable for the treatment type 2 diabetes by improving the sensitivity of muscles or fat to insulin, thereby increasing their glucose uptake. In addition, in order to evaluate the toxic side effects of esculeoside A in the liver, the liver indices, the AST and ALT were evaluated. The results showed that esculeoside A treatment reduced the liver swelling, but did not change the ALT and AST levels. In addition, we found that esculeoside A restored the MDA and TNF- $\alpha$ levels in the liver. These results indicated that esculeoside A might exhibit protective effects on the liver.

Tomato is considered as a healthy food with very low glycemic index, which makes it a healthy food for diabetics [35]. Indeed, tomato consumption has been associated with a reduced risk of chronic non-communicable diseases, including diabetes [9]. However, the epidemiologic studies on the role of tomatoes in prevention of T2DM are limited. It is generally believed that lycopene represents the main bioactive compound in tomatoes. Lycopene is a powerful free radical scavenger and tomatoes are a rich source of lycopene. Recent studies have shown that antioxidants exhibit protective effects against the development of diabetes [36]. A few previous studies have assessed the effects of lycopene on BG levels, lycopene supplementation or lycopene-containing foods appear to exhibit beneficial effects on insulin resistance or 
BG levels in experimental type 2 diabetes model [10, 37, 38]. However, direct evidence with respect to the beneficial effects of lycopene on BG levels is still lacking; moreover, the epidemiologic studies on the association between lycopene or lycopene-containing foods and T2DM are scarce. Many studies have suggested that lycopene may not have a role in the prevention of T2DM $[35,39,40]$. In present study, we demonstrated that tomato main compound esculeoside A possessed anti-hyperglycemic properties, which indicated that the effective component of tomato responsible for hypoglycemic effects is esculeoside A. As far as we know, there is no published study for dietary tomatoes decreased blood sugar in humans. The reason might be that the level of Esculeoside A is very low in dietary tomatoes. In our study, esculeoside A accounted for $0.021 \%$ of dry weight of raw cherry tomatoes; $100 \mathrm{mg} / \mathrm{kg}$ esculeoside A was administered by gavage to the $d b / d b$ mice which reduced the FBG levels. According to the previous literature [41] and the results of this study, if the mouse data is extrapolated to humans, a person must consume $1802 \mathrm{~g}$ of raw tomatoes to obtain enough esculeoside A that could provide adequate beneficial effects by lowering blood glucose. In previous epidemiologic studies, volunteers were not request to consume such high quantity of tomatoes every day. Our findings suggested a possible usefulness of esculeoside A (or TSCE) as a functional supplement for diabetes treatment; however, the possible beneficial effects of esculeoside A in human diabetes need to be further studied.

\section{Conclusions}

These findings suggested that esculeoside A has the potential of alleviating the metabolic abnormalities in diabetic mice via regulation of AMPK/IRS-1 pathway. Our findings also supported a potential role of esculeoside A as a functional supplement for diabetes treatment.

\section{Abbreviations \\ ALT: Alanine aminotransferase; AMPK: Amp-activated protein kinase; \\ AST: Aspartate aminotransferase; FBG: Fasting blood glucose; GCK: Glucokinase; IL-1ß: Interleukin-1 3 ; IL-6: Interleukin-6; INS: Insulin; IPGTT: Intraperitoneal glucose tolerance test; IRS-1: Insulin receptor substrate- 1; IRS-2: Insulin receptor substrate-2; MDA: Malondialdehyde; $\mathrm{p}$ - AMPK: phosphorylated Amp-activated protein kinase; PEPCK: Phosphoenolpyruvate carboxykinase; SOD: Superoxide dismutase; TC: Total cholesterol; TG: Triglycerides; TNF-a: Tumor necrosis factor}

\section{Publisher's Note}

Springer Nature remains neutral with regard to jurisdictional claims in published maps and institutional affiliations.

\section{Acknowledgments}

Thanks for the suggestions and comments of Chung S. Yang, Rutgers, The State University of New Jersey University, Piscataway, NJ, USA.

\section{Authors' contributions}

In this study, CYY and YZM designed the experiments; YZM, ZL, WL and LJ conducted experiments; LDP and LFL contributed reagents, materials, and analytic tools; CYY and YZM performed data analysis and wrote the paper. All authors approved the final manuscript.

\section{Funding}

This work was supported by the Guangxi Science and Technology Innovation Capability and Condition Construction Plan Project (GuiKeNeng 1598025-32), the Guangxi Zhuang Autonomous Region Science and Technology Chairmen Fund (16449-08), and the Guangxi innovation-driven development special project (Guike AA17204038). Funds have been used in areas such as experimentation, analysis and interpretation of data.

\section{Availability of data and materials}

The datasets analyzed during the current study are available from the corresponding author on reasonable request.

\section{Ethics approval and consent to participate}

The mice were supplied by the Model Animal Research Center of Nanjing University (nanjing china) and were treated using the protocol approved by the Institutional Animal Care and Use Committee at the Guangxi Zhuang Autonomous Region and Chinese Academy of Science, Guangxi Institute of Botany (Approval Number: GXZW20161008). Mice were also handled according to the guidelines set by the National Institutes of Health.

\section{Consent for publication}

Not applicable.

\section{Competing interests}

The authors declare that they have no competing interests.

\section{Author details}

${ }^{1}$ Guangxi Key Laboratory of Functional Phytochemicals Research and Utilization, Institute of Botany, Guangxi Zhuang Autonomous Region and Chinese Academy of Sciences, Guilin 541006, China. ${ }^{2}$ Guangxi Institute of Botany, Chinese Academy of Sciences, Road 85 Yanshan, Guilin 541006, China.

Received: 19 March 2019 Accepted: 3 June 2019

Published online: 18 June 2019

\section{References}

1. Pok EH, Lee WJ. Gastrointestinal metabolic surgery for the treatment of type 2 diabetes mellitus. World J Gastroenterol. 2014;20:14315-28.

2. Reddy MA, Zhang E, Natarajan R. Epigenetic mechanisms in diabetic complications and metabolic memory. Diabetologia. 2015;58:443-55.

3. Natarajan R, Nadler JL. Lipid inflammatory mediators in diabetic vascular disease. Arterioscler Thromb Vasc Biol. 2004;24:1542-8.

4. Taylor R. Type 2 diabetes: etiology and reversibility. Diabetes Care. 2013;36: 1047-55.

5. Cho SJ, Park HJ, Jung UJ, Kim HJ, Moon BS, Choi MS. The beneficial effects of combined grape pomace and omija fruit extracts on hyperglycemia, adiposity and hepatic steatosis in $d b / d b$ mice: a comparison with major index compounds. Int J Mol Sci. 2014;15:17778-89.

6. $\quad$ sing LP. The potential protective action of vitamin $D$ in hepatic insulin resistance and pancreatic islet dysfunction in type 2 diabetes mellitus. Nutrients. 2016;8:147-60

7. Matsumoto M, Ogawa W, Teshigawara K, Inoue H, Miyake K, Sakaue H, et al Role of the insulin receptor substrate 1 and phosphatidylinositol 3-kinase signaling pathway in insulin-induced expression of sterol regulatory element binding protein $1 \mathrm{c}$ and glucokinase genes in rat hepatocytes. Diabetes. 2002;51:1672-80.

8. Fryer LG, Carling D. AMP-activated protein kinase and the metabolic syndrome. Biochem Soc Trans. 2005;33:362-6.

9. Raiola A, Rigano MM, Calafiore R, Frusciante L, Barone A. Enhancing the health-promoting effects of tomato fruit for biofortified food. Mediat Inflamm. 2014:5:139873.

10. Li W, Wang G, Lu X, Jiang Y, Xu L, Zhao X. Lycopene ameliorates renal function in rats with streptozotocin-induced diabetes. Int J Clin Exp Pathol. 2014;7:5008-15

11. Fujiwara $Y$, Kiyota $N$, Hori M, Matsushita S, lijima $Y$, Aoki K, et al. Esculeogenin a, a new tomato sapogenol, ameliorates hyperlipidemia and 
atherosclerosis in ApoE-deficient mice by inhibiting ACAT. Arterioscler Thromb Vasc Biol. 2007:27:2400-6.

12. Lu FL, Liu JL, Chen Y, Wu J, Li D. Determination of esculeoside a in Lycopersicon esculentum MILL by high performance liquid chromatography with evaporative light scattering detection. Chin J Chromatogr. 2010;28: 908-11.

13. Lu FL, Chen SC, Liu JL, et al. Separation and purification of total saponins in Lycopersicon esculentum MILL. By D101 macropere resin. Food Sci Technol. 2012;37:199-202.

14. Hummel KP, Dickie MM, Coleman DL. Diabetes, a new mutation in the mouse. Science. 1966;153:1127-8.

15. Kim MJ, Chung JY, Kim JH, Kwak HK. Effects of cranberry powder on biomarkers of oxidative stress and glucose control in $d b / d b$ mice. Nutr Res Pract. 2013;7:430-8.

16. Sotourquieta MG, Lópezbriones S, Pérezvázquez V, Saavedramolina A, Gonzalezhernandez GA, Ramirezemiliano J. Curcumin restores mitochondrial functions and decreases lipid peroxidation in liver and kidneys of diabetic db/db mice. Biol Res. 2014;47:74-82.

17. Fujiwara Y, Takaki A, Uehara Y, Ikeda T, Okawa M, Yamauchi K, et al. Tomato steroidal alkaloid glycosides, Esculeosides a and B, from ripe fruits. Tetrahedron. 2004:60:4915-20.

18. Ju X, Cui Q, Zhang M, Wang W, Jiang J, Chang Y, et al. Human cholesteryl ester transfer protein enhances insulin-mediated glucose uptake in adipocytes. Life Sci. 2011;89:479-84.

19. Zhang $X$, Yang J, Guo Y, Ye H, Yu C, Xu C, et al. Functional proteomic analysis of nonalcoholic fatty liver disease in rat models: Enoyl-coenzyme a hydratase down-regulation exacerbates hepatic steatosis. Hepatology. 2010; 51:1190-9.

20. Liu Q, Li X, Li C, Zhang Y, Wang F, Li H, et al. 1-Deoxynojirimycin alleviates liver injury and improves hepatic glucose metabolism in $d b / d b$ mice. Molecules. 2016;21:279-90.

21. Rocha A, Bolin AP, Cardoso CA, Otton R. Green tea extract activates AMPK and ameliorates white adipose tissue metabolic dysfunction induced by obesity. Eur J Nutr. 2015. https://doi.org/10.1007/s00394-015-1033-8.

22. Narbonne P, Roy R. Caenorhabditis elegans dauers need LKB1/AMPK to ration lipid reserves and ensure long-term survival. Nature. 2009;457:210-4.

23. Hu R, Yan H, Fei X, Liu H, Wu J. Modulation of glucose metabolism by a natural compound from Chloranthus japonicus via activation of AMPactivated protein kinase[J]. Sci Rep. 2017;7:778-87.

24. Zhong J, Gong W, Lu L, Chen J, Lu Z, Li HY, et al. Irbesartan ameliorates hyperlipidemia and liver steatosis in type 2 diabetic $d b / d b$ mice via stimulating PPAR- $y$, AMPK/Akt/mTOR signaling and autophagy. Int Immunopharmacol. 2017:42:176-84.

25. Hu X, Wang $S, X u$ J, Wang DB, Chen $Y$, Yang GZ. Triterpenoid saponins from stauntonia chinensis ameliorate insulin resistance via the AMP-activated protein kinase and IR/IRS-1/PI3K/Akt pathways in insulin-resistant HepG2 cells. Int J Mol Sci. 2014;15:10446-58.

26. Moller DE. New drug targets for type 2 diabetes and the metabolic syndrome. Nature. 2001;414:821-7.

27. Burgess SC, Hausler N, Merritt M, Jeffrey FMH, Storey C, Milde A, et al. Impaired tricarboxylic acid cycle activity in mouse livers lacking cytosolic phosphoenolpyruvate carboxykinase. J Biol Chem. 2004;279:48941-9.

28. Hakimi P, Johnson MT, Yang J, Lepage DF, Conlon RA, Kalhan SC, et al. Phosphoenolpyruvate carboxykinase and the critical role of cataplerosis in the control of hepatic metabolism. Nutr Metab. 2005;2:33-44.

29. Hardie DG. The AMP-activated protein kinase pathway-new players upstream and downstream. J Cell Sci. 2004;1 17:5479-87.

30. Taniguchi CM, Ueki K, Kahn CR. Complementary roles of IRS-1 and IRS-2 in the hepatic regulation of metabolism. J Clin Invest. 2005;115:718-27.

31. Agius L. Glucokinase and molecular aspects of liver glycogen metabolism. Biochem J. 2008;414:1-18.

32. Irwin DM, Tan H. Evolution of glucose utilization: Glucokinase and glucokinase regulator protein. Mol Phylogenet Evol. 2014;70:195-203.

33. Nathan DM, Davidson MB, Defronzo RA, Heine RJ, Henry RR, Pratley R, et al. Impaired fasting glucose and impaired glucose tolerance implications for care. J Am Coll Cardiol. 2007. https://doi.org/10.2337/dc07-9920.

34. Poovitha S, Parani M. Protein extract from the fruit pulp of Momordica charantia potentiate glucose uptake by up-regulating GLUT4 and AMPK. J Funct Foods. 2017;37:507-12.

35. Shidfar F, Froghifar N, Vafa M, Rajab A, Hosseini S, Shidfar S, et al. The effects of tomato consumption on serum glucose, apolipoprotein $\mathrm{B}$, apolipoprotein
A-I, homocysteine and blood pressure in type 2 diabetic patients. Int J Food Sci Nutr. 2011;62:289-94.

36. Montonen J, Knekt P, Järvinen R, Reunanen A. Dietary antioxidant intake and risk of type 2 diabetes. Diabetes Care. 2004:27:362-6.

37. Zidani S, Benakmoum A, Ammouche A, Benali Y, Bouhadef A, Abbeddou S. Effect of dry tomato peel supplementation on glucose tolerance, insulin resistance, and hepatic markers in mice fed high-saturated-fat/highcholesterol diets. J Nutr Biochem. 2016;40:164-71.

38. Ali MM, Agha FG. Amelioration of streptozotocin-induced diabetes mellitus, oxidative stress and dyslipidemia in rats by tomato extract lycopene. Scand J Clin Lab Invest. 2009;69:371-9.

39. Wang L, Liu S, Manson JE, Gaziano LM, Buring JE, Sesso HD. The consumption of lycopene and tomato-based food products is not associated with the risk of type 2 diabetes in women. J Nutr. 2006;136:620-5.

40. Upritchard JE, Sutherland WHF, Mann JI. Effect of supplementation with tomato juice, vitamin $E$, and vitamin C on LDL oxidation and products of inflammatory activity in type 2 diabetes. Diabetes Care. 2000;23:733-8.

41. Reaganshaw S, Nihal M, Ahmad N. Dose translation from animal to human studies revisited. FASEB J. 2008;22:659-61.
Ready to submit your research? Choose BMC and benefit from:

- fast, convenient online submission

- thorough peer review by experienced researchers in your field

- rapid publication on acceptance

- support for research data, including large and complex data types

- gold Open Access which fosters wider collaboration and increased citations

- maximum visibility for your research: over $100 \mathrm{M}$ website views per year

At BMC, research is always in progress.

Learn more biomedcentral.com/submissions 